\title{
Phonon Rabi-assisted tunneling in diatomic molecules
}

\author{
E. Vernek, ${ }^{1,2}$ E. V. Anda, ${ }^{2}$ S. E. Ulloa,${ }^{1}$ and N. Sandler ${ }^{1}$ \\ ${ }^{1}$ Department of Physics and Astronomy, and Nanoscale and Quantum Phenomena Institute, \\ Ohio University, Athens, Ohio 45701-2979 \\ ${ }^{2}$ Departamento de Física, Pontifícia Universidade Católica, Rio de Janeiro-RJ, Brazil
}

(Dated: 21 May 2005)

\begin{abstract}
We study electronic transport in diatomic molecules connected to metallic contacts in the regime where both electron-electron and electron-phonon interactions are important. We find that the competition between these interactions results in unique resonant conditions for interlevel transitions and polaron formation: the Coulomb repulsion requires additional energy when electrons attempt phonon-assisted interlevel jumps between fully or partially occupied levels. We apply the equations of motion approach to calculate the electronic Green's functions. The density of states and conductance through the system are shown to exhibit interesting Rabi-like splitting of Coulomb blockade peaks and strong temperature dependence under the interacting resonant conditions.

PACS numbers: 73.23.-b, 73.63.Kv, 71.38.- $\mathrm{k}$
\end{abstract}

A significant current effort in nanoscopic systems is the study of electron transport in natural and quantumdot molecules. Much of the interest lies in being able to investigate different regimes of competing electronelectron and electron-phonon interactions. It is typically the case, due to the spatial confinement, that electronelectron interactions (EEI) play a more important role than electron-phonon interactions (EPI) in determining electronic transport properties in low-dimensional systems.

Different geometries of quantum-dot molecules (constructed with interconnected quantum dots) have been studied in the literature, and the role of phonons on electron transport has been analyzed in these systems. $\frac{1}{1}$ For instance, it is known that phonons are a relatively weak perturbation, responsible for the broadening of Coulomb blockade peaks in the conductance and for the appearance of satellite features in the nonlinear transport regime $\frac{1}{n}$

More recently, the field of "molecular electronics," 2.3 where electrons and/or holes are injected directly into molecules attached to metal electrodes, has seen intense activity and progress ${ }^{4.5}$ It is interesting to note that EPI become more important in molecular electronics, since local molecule deformations produce significant electronic level shifts, as has been observed in experiments ${ }^{6}$ In fact, vibrational and torsional modes play prominent roles in electron transport, producing sidebands in the voltagedependent differential conductance $\frac{7.8 .9}{}$ and/or polaronic shifts of the electronic levels $\stackrel{10}{\underline{10}}$ Furthermore, in a molecular system with discrete electronic energy levels, vibrations produce important effects when the energy of the vibrational modes matches the energy difference between electronic levels 11 As a result, EPI provides relaxation mechanisms (inelastic scattering) that affect the conductance of the system: ${ }^{12}$

The simplest model of EPI is perhaps the independent boson model, ${ }^{13}$ where localized electrons interact with a phonon system. Phonons introduce a shift of electronic levels and create a series of phonon replica peaks in the density of states ( DOS) of the electronic system. In a double barrier heterostructure interacting with phonons, as electrons have access to an energy continuum in the region outside the barriers, the inelastic scattering strongly affects the resonant tunneling regime through the heterostructure ${ }^{14}$ The effects of EPI on a double-

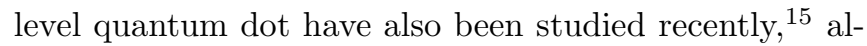
though the combined effects of inelastic scattering and EEI were not considered.

In order to study a more realistic molecular system it is important to include both interactions - EEI and EPI - simultaneously. The interplay between the competing interactions is likely to result in unique conditions for phonon emission and absorption, as well as in unexpected polaron behavior ${ }^{10}$ In this work we study the effect that EEI and EPI have on charge transport through a diatomic molecule, envisioned as two atomic sites (or quantum dots) directly coupled to leads, as shown schematically in Fig. 10 We study this system using the equations of motion method, which allows us to obtain the DOS and electronic occupation as well as the conductance through the system. We exploit the fact that the EPI strength $\lambda$ is small compared to the phonon energy, and thus include self-energy terms up to second order in this parameter. Standard considerations, similar to those applied in the Hubbard approximation, ${ }^{16.17}$ are used to evaluate the equations. A significant result is the identification of occupation-dependent resonant conditions for phonon absorption and emission in the presence of Coulomb repulsion. More importantly, we find a unique type of Rabi splitting in the DOS from the mixing between a doubly occupied low-energy level (boosted by the Coulomb repulsion) and a higher-energy state. This Rabi splitting is mediated by thermally regulated phonon emission and absorption in the molecule. The effect is shown to dramatically modify the transport properties of the system since the resulting polaron formation competes with resonant tunneling. The effect is made remarkably noticeable even for weak EPI since the phonon-assisted transport is magnified by the virtual emission and absorption pro- 


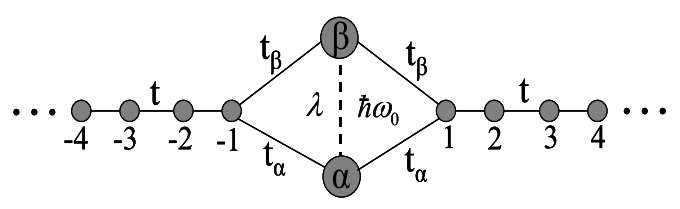

FIG. 1: Schematic representation of the model system. The electron-phonon interaction connects the local sites $\left(\epsilon_{\alpha}<\epsilon_{\beta}\right)$ via phonons of frequency $\omega_{0}$ with coupling constant $\lambda$.

cesses in the interacting resonant regime.

For concreteness we consider a two-level diatomic molecule with local energies $\epsilon_{\alpha}^{0}$ and $\epsilon_{\beta}^{0}$ (we assume $\epsilon_{\beta}^{0}>$ $\left.\epsilon_{\alpha}^{0}\right)$, as shown in Fig. 1] Each dot or atomic site is connected independently to two external current leads. The system as described can be mapped to that studied in Ref. 15. (there for the one-electron case) and is designed to model various experimental geometries. The total Hamiltonian is written as $H_{T}=H_{\text {mol }}+H_{\text {leads }}+$ $H_{\text {mol-leads }}$. Each lead is modeled as a semi-infinite tightbinding chain, $H_{l e a d}=\sum_{\sigma,<j^{\prime}, j>} t c_{j^{\prime} \sigma}^{\dagger} c_{j \sigma}$, where the site index sum is over nearest neighbors, $c_{j \sigma}^{\dagger}\left(c_{j \sigma}\right)$ creates (annihilates) a fermion at the $j$-th site with spin $\sigma$, and the left (right) lead is defined for $j^{\prime}, j \leq-1$ $\left(j^{\prime}, j \geq 1\right)$. The Hamiltonian for the molecule is given by $H_{m o l}=H_{e l}+H_{p h}+H_{e l-p h}$,

$$
\begin{aligned}
H_{e l} & =\sum_{\sigma, i=\alpha, \beta}\left[\epsilon_{i} c_{i \sigma}^{\dagger} c_{i \sigma}+\frac{U}{2} n_{i \sigma} n_{i \bar{\sigma}}\right], \\
H_{p h} & =\left(b^{\dagger} b+\frac{1}{2}\right) \hbar \omega_{0},
\end{aligned}
$$

and in the rotating-wave approximation ${ }^{13}$

$$
H_{e l-p h}=\lambda \sum_{\sigma}\left(b^{\dagger} c_{\alpha \sigma}^{\dagger} c_{\beta \sigma}+H . c\right)
$$

where $b^{\dagger}(b)$ creates (annihilates) a phonon with energy $\hbar \omega_{0}, \epsilon_{i}=\epsilon_{i}^{0}-e V_{g}$, and $i=\alpha, \beta$. The gate voltage $V_{g}$ controls the particle number by shifting both localized energies with respect to the Fermi energies of the left and right electrodes. We assume here the same gate on both sites, as is likely the case in molecules ${ }^{6}$ Finally, $H_{\text {mol-leads }}=\sum_{\substack{\sigma, i=\alpha, \beta \\ j=1,-1}} t_{i} c_{i \sigma}^{\dagger} c_{j \sigma}+H . c$. , connects the diatomic molecule to the leads, where we will consider $t_{\alpha}, t_{\beta} \ll t$. Away from the Kondo regime the effect of the leads is to broaden the energy levels of the dots through the tunnel couplings $t_{\alpha}$ and $t_{\beta}$. (Ref. 18)

To determine the dynamics of the electrons in the molecule, we calculate the local retarded Green's function, together with the nonlocal functions,

$$
i G_{\beta \alpha}^{\sigma}(\epsilon)=\int \theta(t)\left[\begin{array}{c}
\left\langle\left[b c_{\alpha \sigma}(t), c_{\beta \sigma}^{\dagger}(0)\right]_{+}\right\rangle \\
\left\langle\left[b^{\dagger} c_{\beta \sigma}(t), c_{\alpha \sigma}^{\dagger}(0)\right]_{+}\right\rangle
\end{array}\right] e^{i \epsilon t} d t .
$$

The latter are needed since they describe electron propagation due to the EPI, and are associated with the absorption $\left(G_{\alpha \beta}^{\sigma}\right)$ and emission $\left(G_{\beta \alpha}^{\sigma}\right)$ of phonons. The corresponding equations of motion up to second order in the EPI coupling strength $\lambda$, yield $(i=\alpha, \beta)$,

$$
\begin{array}{r}
G_{i i}(\epsilon)=\frac{\epsilon}{\left(\epsilon-\epsilon_{i}\right)\left(\epsilon-\epsilon_{i}-U\right)} \\
G_{\beta \alpha}(\epsilon)=\frac{\lambda\left[\left\langle b^{\dagger} b\right\rangle+\left\langle n_{\beta}\right\rangle\right] G_{\alpha \alpha}(\epsilon)}{\epsilon-\epsilon_{\beta}-U\left\langle n_{\beta}\right\rangle+\hbar \omega_{0}+i \Gamma\left(\epsilon+\hbar \omega_{0}\right)}, \\
G_{\alpha \beta}(\epsilon)=\frac{\lambda\left[\left\langle b^{\dagger} b\right\rangle+1-\left\langle n_{\alpha}\right\rangle\right] G_{\beta \beta}(\epsilon)}{\epsilon-\epsilon_{\alpha}-U\left\langle n_{\alpha}\right\rangle-\hbar \omega_{0}+i \Gamma\left(\epsilon-\hbar \omega_{0}\right)},
\end{array}
$$

where the expressions for the corresponding self-energies are given by

$$
\begin{aligned}
& \Sigma_{\alpha}=\lambda^{2} \frac{\left[\left\langle b^{\dagger} b\right\rangle+\left\langle n_{\beta}\right\rangle\right]\left(\epsilon-\epsilon_{\alpha}-U\right)}{\epsilon-\epsilon_{\beta}-U\left\langle n_{\beta}\right\rangle+\hbar \omega_{0}+i \Gamma\left(\epsilon+\hbar \omega_{0}\right)}, \\
& \Sigma_{\beta}=\lambda^{2} \frac{\left[\left\langle b^{\dagger} b\right\rangle+1-\left\langle n_{\alpha}\right\rangle\right]\left(\epsilon-\epsilon_{\beta}-U\right)}{\epsilon-\epsilon_{\alpha}-U\left\langle n_{\alpha}\right\rangle-\hbar \omega_{0}+i \Gamma\left(\epsilon-\hbar \omega_{0}\right)} .
\end{aligned}
$$

We have considered here the paramagnetic case with $\left\langle n_{i \bar{\sigma}}\right\rangle=\left\langle n_{i \sigma}\right\rangle \equiv\left\langle n_{i}\right\rangle$. The broadening due to the leads is

given by $\Gamma_{i}(\epsilon)=2 \pi t_{i}^{2} \rho(\epsilon)$, where $\rho(\epsilon)$ is the DOS in the leads. ${ }^{1}$ We take $\Gamma_{\alpha}=\Gamma_{\beta}=\Gamma(\epsilon)$, for simplicity $\underline{19}$

The nonlocal Green's functions, Eqs. (6) and (7), have simple physical interpretations. At low temperature $T$, $\left\langle b^{\dagger} b\right\rangle \approx 0$ thus $G_{\beta \alpha} \rightarrow 0$ as $\left\langle n_{\beta}\right\rangle \rightarrow 0$, i.e., there is no phonon emission if the $\beta$ level is empty. It is also interesting to note that if the $\operatorname{dot} \alpha$ is not completely full, $\left\langle n_{\alpha}\right\rangle<1$, the process of phonon absorption described by $G_{\alpha \beta}$ is possible (even at small $T$ ).

In the limit $U=0$ the local Green's functions $G_{i i}$ have two poles, one at $\epsilon_{i}$ and another at the pole of the self-energy $\Sigma_{i}$. In this case there is a single phonon resonance condition for both levels $(i=\alpha, \beta)$, achieved when the phonon energy $\hbar \omega_{0}$ matches the energy difference between the two localized electron energies $\Delta \epsilon \equiv \epsilon_{\beta}-\epsilon_{\alpha}$, 


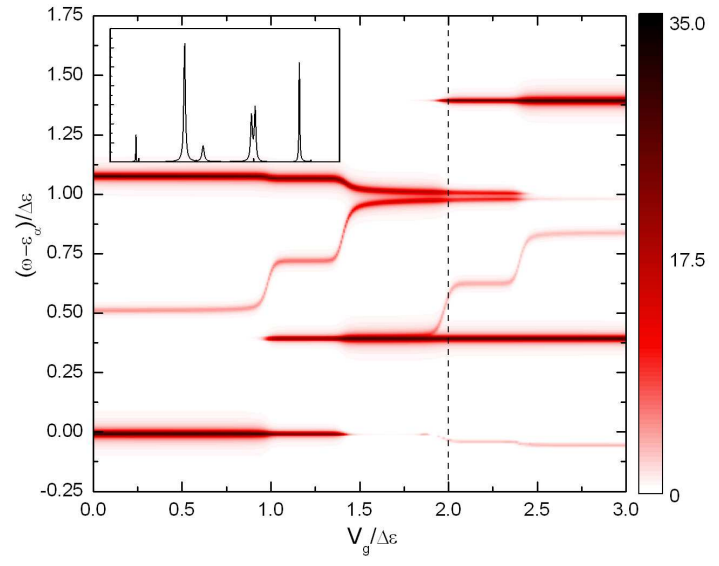

FIG. 2: (Color online) Total density of states (DOS) vs energy and gate voltage for a two-site molecule as in Fig. 1] In units of $\Delta \epsilon$, the parameters are: $\lambda=0.2, k_{B} T=0.015$, $t_{\alpha}=t_{\beta}=0.1, U=0.4$, and $\hbar \omega_{0}=0.6$. The Fermi level is set at zero energy; $\epsilon_{\alpha}=1$ and $\epsilon_{\beta}=2$ for $V_{g}=0$. For $V_{g} \approx 1.6-$ 2.4 , the DOS exhibits a clearly split feature at $\omega-\epsilon_{\alpha} \approx 1$ produced by the strong phonon-assisted level coupling. The inset shows the DOS at $V_{g}=2$, indicated by the dashed line in the main figure.

i.e., at $\Delta \epsilon=\hbar \omega_{0}$. Thus, the resonance effectively couples both sites. Such a phonon resonance condition on transport has been recently explored by Tasai and Eto 15 They find a sharp dip in the conductance as the result of destructive interference between bonding and antibonding states (see the dashed line in Fig. 2). We will see below that electron repulsion greatly affects this behavior.

In the limit $\lambda=0$ we recover the Hubbard I approximation ${ }^{16}$ As expected, each local Green's function has poles at $\epsilon_{i}$ and $\epsilon_{i}+U$. In contrast, when $U \neq 0$, we find different resonant conditions for $\alpha$ and $\beta$. The Coulomb repulsion requires extra energy for electrons to tunnel into a fully or partially occupied state. This extra energy depends on the occupation fraction of the two sites, which can be controlled by the gate voltage. The self-consistent charge of each site is obtained by integrating the DOS, $\rho_{i i}=(-1 / \pi) \operatorname{Im} G_{i i}(\epsilon)$, for different gate voltages. With appropriate parameter values we find that the effects brought about by the EPI and EEI are emphasized under resonant conditions. Hereafter all energy quantities are given in units of the level spacing $\Delta \epsilon$.

Figure 2 shows the total (for both sites) DOS for the molecular system as a color map for different values of gate voltage $V_{g}$ and energy $\omega$, measured with respect to the lowest level $\epsilon_{\alpha}$. The temperature is set at $k_{B} T=$ $0.015, U=0.4$, and $\hbar \omega_{0}=0.6$. [The DOS is normalized to obtain the correct charge number for each dot.] For low-voltage values, the molecule is empty and the DOS shows two main features at the noninteracting energies $\omega \simeq \epsilon_{\alpha}, \epsilon_{\beta}$, slightly shifted and broadened by the coupling to the phonons and leads. As the gate voltage increases, the lowest level approaches the Fermi level, and as its occupancy grows a unique feature in the DOS develops.

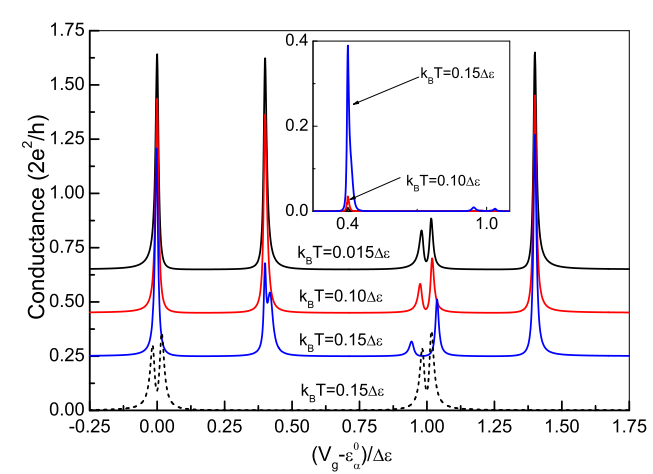

FIG. 3: (Color online) Conductance vs gate voltage for $k_{B} T / \Delta \epsilon=0.015,0.10$, and 0.15 . Temperature has a strong effect on the resonance condition, enhancing the Rabi splitting of the third Coulomb blockade peak $\left(V_{g}-\epsilon_{\alpha} \simeq 1\right)$ at higher $T$ and exposing the shoulder in second peak. The inset shows inelastic contributions for the conductance. The highest peak is due to phonon emission. The dashed line shows results for $U=0$ and $\omega_{0}=1$. Curves offset for clarity.

This is shown in Fig. 2 at energy $\epsilon_{\alpha}+U \approx 0.4$ for $V_{g} \gtrsim$ 1. Notice that at the same time, a weak phonon-related feature (a "phonon replica") appears at energy $\simeq \epsilon_{\alpha}+$ $\hbar \omega_{0}=0.6$, as can be seen from the expression for the self-energy, Eq. (9). [This feature persists weakly even at low $V_{g}$ at $\simeq 0.5$, due to the finite $\lambda$ and $T$ values in Fig. [2]

For $V_{g} \gtrsim 1.4$, the lowest level is almost fully occupied, $\left\langle n_{\alpha}\right\rangle \simeq 1$, and the resonant absorption/emission condition in Eq. (9) is achieved when $\epsilon_{\alpha}+U\left\langle n_{\alpha}\right\rangle+\hbar \omega_{0} \simeq \epsilon_{\beta}$. The increasing $\left\langle n_{\alpha}\right\rangle$ occupation shifts the phonon replica feature, moving it closer to $\epsilon_{\beta}$. The resonant condition results in the effective mixing of the $\epsilon_{\alpha}+U$ and $\epsilon_{\beta}$ levels due to phonon-assisted transitions. The mixing produces a near degeneracy and, as a consequence, an effective phonon Rabi splitting of spectral features appears. This is the origin of the doublet appearing at $\omega-\epsilon_{\alpha} \simeq 1$ with nearly equal-size peaks in the range $V_{g} \approx 1.6-2.4$. Note that the lower level participates in the process as a result of the Coulomb repulsion between electrons. Notice further that the resonant condition disappears once the higher level becomes fully (doubly) occupied $\left(V_{g} \gtrsim 2.4\right.$ in Fig. 2). The DOS returns then to the standard Hubbard peaks at $\epsilon_{\alpha}+U$ and $\epsilon_{\beta}+U$ dominating the spectrum of the molecule (weak phonon-related features near 0 and 0.8 are present for finite $T$ and $\lambda$ ). As we will show below, the Rabi resonance in the presence of EEI affects also the conductance through the system, providing an experimentally accessible signature of the effect.

Figure 3 shows the conductance $G$ vs gate voltage for different temperatures $\left(G \propto\left|G_{\overline{1} 1}\right|^{2}\right.$ where $G_{\overline{1} 1} \propto$ $\left.t_{\alpha}^{2} G_{\alpha \alpha}+t_{\beta}^{2} G_{\beta \beta}+t_{\alpha}\left[G_{\alpha \beta}+G_{\beta \alpha}\right] t_{\beta}\right)$. As the gate voltage increases, the conductance exhibits the anticipated Coulomb blockade $(\mathrm{CB})$ peaks. Notice that the first two are associated here with $\epsilon_{\alpha}$, and show nearly full $e^{2} / h$ 


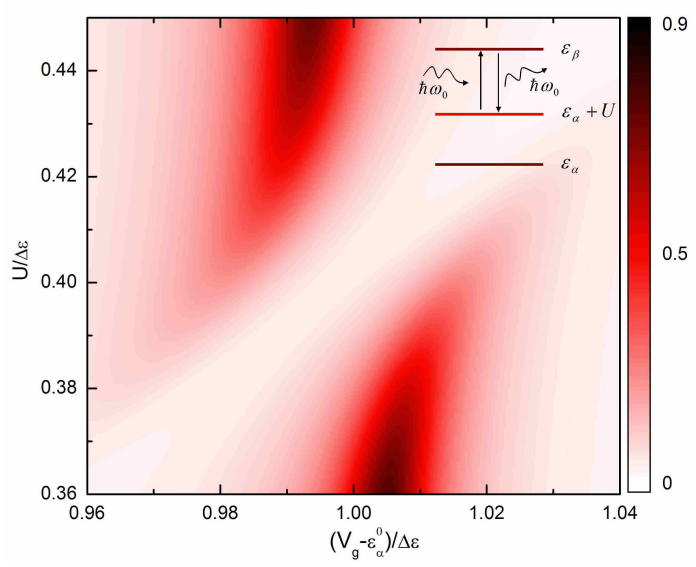

FIG. 4: (Color online) Conductance through the molecule at around the third Coulomb blockade peak as a function of interaction $U$ and gate voltage $V_{g}$ for $k_{B} T=0.015 \Delta \epsilon$. The inset indicates phonon emission and absorption processes involved.

conductance (limited by finite temperatures). The dominant effect of higher temperatures is to produce important changes in the dot occupancy due to phonon emission (occupancy changes due to thermal excitation of electrons are negligible). The third CB peak appearing at $V_{g}-\epsilon_{\alpha}^{0} \simeq 1$ is clearly split, indicating the phononmediated transitions between the $\alpha$ and $\beta$ levels. The temperature dependence of this peak is pronounced, as the thermal variation in electron and phonon occupations will not only make it weaker but will also rapidly detune the resonance condition. A higher phonon presence results in stronger EPI and a drop in the conductance, a typical signature of interference between resonant tunneling and polaron formation processes. The splitting of the third CB peak increases linearly with the EPI strength $\lambda$, as expected in Rabi splitting phenomena. Notice that once the $\beta$ level is nearly full, the effect disappears, and the Coulomb blockade peak at $V_{g}-\epsilon_{\alpha}^{0} \simeq U+\Delta \epsilon=1.4$ is essentially $T$ independent. The appearance of a shoulder in the second $\mathrm{CB}$ peak for higher temperatures is also a consequence of EPI, but one that is inelastic in nature: The inset of Fig. B shows the contribution of inelastic processes $\left(\propto G_{\alpha \beta}+G_{\beta \alpha}\right)$ to the conductance. The highest peak at $V_{g}-\epsilon_{\alpha}^{0} \simeq 0.4$ is due to phonon emission processes: electrons thermally excited to level $\epsilon_{\beta}$ (above the Fermi level here) fall to level $\epsilon_{\alpha}+U$ by the emission of phonons (the inset of Fig. प पillustrates levels involved). These processes do not enhance the conductance of the resonant level, but rather reduce it, as one sees in the main panel. The suppression is produced by the destructive interference of the two different conducting processes that electrons undergo.

The resonant condition of phonon-assisted tunneling through the molecule in the presence of EEI is a function of the interaction parameter $U$ and the occupation of the molecule. In order to explore the dependence of the Rabi splitting on interaction parameters we show in Fig. 4 a color map of the conductance as function of $U / \Delta \epsilon$ and $\left(V_{g}-\epsilon_{\alpha}^{0}\right) / \Delta \epsilon$. It is clear that the conductance returns to the non-phonon-assisted resonant single $\mathrm{CB}$ peak whenever $U / \Delta \epsilon$ is far below or above the resonant value $U \simeq \Delta \epsilon-\hbar \omega_{0}=0.4$. The lowest level is nearly doubly occupied in this $V_{g}$ regime, i.e., $\left(V_{g}-\epsilon_{\alpha}^{0}\right) / \Delta \epsilon \simeq 1$. At the resonance, $U \simeq 0.4$, the conductance Rabisplits into two peaks that have the same height and demonstrates the effect of polaron formation on the conductance.

We have shown that the competition of EEI and EPI in a diatomic molecule produces unexpected Rabi splitting phenomena in the DOS with observable effects on the conductance. This phenomenon involves states produced by the Coulomb repulsion, and it is enhanced at higher temperatures, a direct consequence of the thermal nature of the phonon bath involved.

We would like to thank CAPES (Brazil) and NSF-IMC grant No 0336431 for support, and C. Büsser for helpful discussions.
1 W. G. van der Wiel, S. De Franceschi, J. M. Elzerman, T. Fujisawa, S. Tarucha, and L. P. Kouwenhoven, Rev. Mod. Phys. 75, 1 (2003).

${ }^{2}$ L. Kouwenhoven, Science 268, 1440 (1995).

3 J. R. Heath and M. A. Ratner, Phys. Today 56, 43 (2003).

4 Y. García, J. J. Palacios, E. San Fabián, J. A. Vergés, A. J. Pérez-Jiménez, and E. Louis, Phys. Rev. B 69, 041402(R) (2004).

5 R. H. M. Smit, Y. Noat, C. Untiedt, N. D. Lang, M. C. Van Hermet, and J. M. Van Ruitenbeek, Nature 419, 906 (2002).

${ }^{6}$ L. H. Yu, Z. K. Keane, J. W. Ciszek, L. Cheng, M. P. Stewart, J. M. Tour, and D. Natelson, Phys. Rev. Lett. 93, 266802 (2004); J. Park, A.N. Pasupathy, J.I. Goldsmith, C. Chang, Y. Yaish, J.R. Petta, M. Rinkowski, J.P. Sethna, H.D. Abruña, P.L. McEuen, and D.C. Ralph, Nature 417,
722 (2002).

7 H. Park, J. Park, A. K. L. Lim, E. H. Anderson, A. Paul Alivisatos, and P. L. McEuen, Nature 407, 57 (2000).

${ }^{8}$ K. Flensberg, Phys. Rev. B 68, 205323 (2003).

${ }^{9}$ W. Zhang, A. O. Govorov, and S. E. Ulloa, Phys. Rev. B 66, 060303(R) (2002).

10 A. Mitra, I. Aleiner, and A. J. Millis, Phys. Rev. B 69, 245302 (2004).

11 M. I. Vasilevskiy, E. V. Anda and S. S. Makler, Phys. Rev. B 70, 035318 (2004).

12 P. Orellana, E. Anda, and F. Claro Phys. Rev. Lett. 79, 1118 (1997).

13 G. D. Mahan, Many-Particle Physics, 2nd ed. (Plenum, New York, 1990)

14 E. V. Anda and F. Flores, J. Phys. Cond. Mat 3, 9087 (1991). 
15 T. Tasai and M. Eto, Physica E 17, 139 (2003).

16 J. Hubbard, Proc. R. Soc. A 276, 238 (1963).

17 D. N. Zubarev, Usp. Fiz. Nauk 71, 71 (1960).

18 Electron correlations with the leads are not considered in the present work. Thus, the physics described is in the regime $T>T_{K}$, where $T_{K}$ is the Kondo temperature.

$19 U$-dependent vertex renormalization of $\Gamma_{i}$ [e.g., A. Levy
Yeyati, A. Martín-Rodero, and F. Flores, Lect. Notes in Physics 547, 27 (2000)] may introduce minor effects. We tested implementation by W. Meztner [Phys. Rev. B 43, 8549 (1991)] and found no appreciable changes of the results shown here (for $\Delta \epsilon \gg \Gamma$ ). 